Aus dem pathologiseh-anatom. Institut (Prof. Beneke) und der medizin. Klinik (Prof. Brauer) zu Marburg.

\title{
Der Einfluss des künstlichen Pneumothorax auf die tuberkulöse Lunge.
}

(Pathologisch-anatomische Untersuchung.)

Von

Dr. Fr. Graetz,

I. Assistenten am patholog. Institut.

Mit 3 lithograph. Tafeln.

Es ist das Verdienst Forlaninis (1), das Prinzip der Ruhigstellung, wie es in der Chirurgie häufig bei der Behandlung tuberkulöser Gelenkerkrankungen Anwendung findet, auf die tuberkulöse Lunge übertragen zu haben. Schon im Jahre 1882 hatte genannter Autor den Gedanken ausgesprochen, unter Nachahmung natürlicher Vorgänge, den Pneumothorax therapeutisch zu verwerten. Forlanini hat mit diesem Vorschlag die bis dahin ziemlich allgemein gültige Anschauung durchbrochen, dass der Pneumothorax als eine absolut tödliche Komplikation der Lungentuberkulose anzusehen sei.

Auf dem medizinischen Kongress zu Rom im Jahre 1894 berichtete Forlanini über eine Reihe von Fällen, bei denen es im Anschluss an die Entstehung eines Pneumothorax zur Ausheilung der Tuberkalose gekommen war. Auf Grund dieser Beobachtungen nahm Forlanini Veranlassung, den Preumothorax zur Therapie der Lungenphthise dringend $\mathrm{zu}$ empfehlen, und dieses im Laufe des folgenden Jahres auch praktisch auszuführen.

Unterdessen hatten die Beobachtungen Forlaninis auch von anderen Autoren eine wiederholte Bestätigung erfahren. So hatte $\mathrm{Späth}(2)$ bei schweren Fällen progredienter Lungenphthise beim Eintritt eines Pneumothorax wiederholt einen auffallenden Umschwung in den klinischen Erscheinungen, sowie eine günstige Beeinflussung des Grundleidens feststellen können. Auch er führt die Besserung 
und Ausheilung des tuberkulösen Prozesses, die er namentlich in drei Fällen beobachten konnte, auf die Kompression der erkrankten Lunge zurück und rät auch seinerseits, den Pneumothorax der Therapie nutzbar zu machen.

In seiner Abhandlung über die Pleuraempyeme betont Bäumler (3) die "hemmende Wirkung" von Flüssigkeits- oder Luftergüssen in die Pleura und erhofft von ihnen eine günstige Beeinflussung des tuberkulösen Prozesses.

Der Gedanke, dass gerade in den Flüssigkeitsergüssen der heilende Faktor für die Lungentuberkulose zu erblicken sei, findet auch bei Lucius Spengler (4) Ausdruck. An seinem grossen Material hatte Lucius Spengler (5) in einer Reihe von Fällen, über die er in verschiedenen Arbeiten eingehender berichtet, Gelegenheit, den günstigen Einfluss von Pleuraexsudaten zu beobachten und konnte sogar in einem Teil seiner Fälle eine vollkommene Ausheilung des tuberkulösen Prozesses feststellen. Spengler hält es deshalb für direkt ratsam, Pleuraexsudate zu erzeugen.

Ähnliche Beobachtungen, wie wir sie in den deutschen Arbeiten niedergelegt finden, enthält auch zum Teil die ausländische Literatur. So erkennen namentlich Adams (6) und Gailliard (7) sowohl Pleuraergüssen wie dem Pneumothorax eine heilende Wirkung auf die krankhaften Prozesse der Lunge zu.

Der amerikanische Chirurg Murphy (9) hat dann anscheinend unabhängig ron $\mathrm{F}$ orlanini die gleichen Konsequenzen aus ähnlichen Beobachtungen gezogen und den Gedanken in grossem Massstabe in die Tat umgesetzt. Auch er war auf Grund ähnlicher Beobachtungen wie die genannten Autoren zu dem Schlusse gekommen, den Pneumothorax therapeutisch zu verwerten. Massgebend waren für Murphy dabei die Erfolge gewesen, die die Chirurgie bei der Immobilisierung tuberkulöser Gelenke aufzuweisen hatte. Die erste Mitteilung berichtet zunächst über fünf Fälle, die unter Anwendung des Pneumothorax günstig beeinflusst worden seien. Auch bei weiteren Fällen, die, nach einer brieflichen Mitteilung Murphys an Brauer ${ }^{1}$ mit der Injektionsmethode behandelt worden waren, soll das Resultat ein durchaus befriedigendes gewesen sein. Vor allem erfuhren die schweren Erscheinungen wie Fieber und Blutungen eine auffallend schnelle Beeinflussung unter gleichzeitiger Besserung des Allgemeinbefindens.

Soweit. aus der provisorischen Mitteilung dieser Fälle durch Lemke (8) ein Schluss gezogen werden kann, muss man auch hier

1) Cf. die Arbeit von Mosheim, Beiträge z. Klinik der Tuberkul. Bd. III. Heft 5. - Brauer hat in dieser breit angelegten Dissertation die damalige Pneumothorax-Literatur zusammenstellen lassen. 
den Eindruck gewinnen, dass in den 53 von Lemke mit Lungenkompression behandelten Fällen der tuberkulöse Prozess eine Wendung zur Ausheilung nahm.

Neuerdings haben nun Brauer (9) und L. Spengler der Pneumothoraxbehandlung Eingang in Deutschland verschafft. Der Wunsch, auch Phthisikern fortgeschrittener Stadien eine aktive Therapie angedeihen zu lassen, veranlassten die genannten Autoren, angesichts der günstigen Erfolge Murphys u. a., die Anwendung des künstlichen Pneumothorax in grossem Stile zu versuchen. Brauer (10) wandte zunächst das Verfabren bei vier Fällen von fortgeschrittener Lungenphthise sowie bei einigen Fällen mit ausgedehnten Bronchiektasien an. Es lässt sich nicht in Abrede stellen, dass bei einem Teil der Fälle eine günstige Beeinflussung des Befindens erreicht wurde.

In Gemeinschaft mit L. Spengler hat nun B rau er bei 60 Phthisikern der verschiedensten Stadien den therapeutischen Pneumothorax angelegt. Diese Fälle werden demnächst eine eingehende Würdigung in Brauers "Beiträgen zur Klinik der Tuberkulose" finden. Ich selbst hatte Gelegenheit, in persönlicher Besprechung der Fälle mit Herrn Prof. Brauer sowie durch Kenntnisnahme der Krankengeschichten mich von dem günstigen Erfolge der Behandlungsmethode zu überzeugen. Patienten, deren Leiden bis dahin jeglicher Therapie getrotzt hatte, die nach dem Charakter ihre Erkrankung sicher innerhalb kurzer Zeit dem Tode verfallen wären, zeigen heute das beste Wohlbefinden. Der Prozess selbst ist zum Stillstand gekommen, das Allgemeinbefinden ist gut, Fieber und Auswurf sind verschwunden.

Veranlasst durch die Publikationen Bra u ers hat in neuester Zeit auch Forlanini (1) nach längerem Schweigen wieder Stellung gur Pneumothoraxfrage genommen. Auch er ist in der Lage, über eine Anzahl von Fällen zu berichten, bei denen es zu einer Dauerheilung des tuberkulösen Prozesses gekommen ist. Er berichtet über Fälle, bei denen es sich um eine Zeit bis zu 10 Jahren nach Anlegung des Pneumothorax handelt. Besonders bemerkenswert ist die Tatsache, dass es bei verschiedenen Fällen, bei denen durch Pneumothoraxbehandlung eine Ausheilung der komprimierten Lunge eintrat, auch eine völlige Ausheilung geringer Krankheitsprozesse der sonst gesunden anderen Lunge zustande kam. Auch Brauer und L. Spengler konnte günstige Beeinflussung leichter Prozesse auf der anderen, sonst gesunden Lunge beobachten. In einem Falle, bei dem die Antopsie möglich gewesen war, fand sich eine vollkommene schwielige Umwandlung der komprimierten Lunge.

Während die klinischen Verhältnisse beim künstlichen Pneumothorax zum Teil eine eingehende Würdigung erfahren haben, sind die 
anatomischen Untersuchungen bisher bedeutend zu kurz gekommen. Es muss angesichts der grossen Anzahl von Fällen, in denen es zur Anlegung eines künstlichen Pneumothorax gekommen ist, auffallen, dass so gut wie keine Untersuchungen darüber vorliegen, in welcher Weise die langdauernde Kompression der tuberkulösen Lunge das anatomische Bild beeinflusst. Und doch sollte man glauben, dass es nahe gelegen hätte, den Einfluss einer langdanernden Kompression auf die tuberkulöse Lunge anatomisch zu prüfen, zumal ja davon die Bewertung des künstlichen Pneumothorax als einer kausalen Behandlungsmethode abhängig gemacht werden muss. Worin diese Nichtbeachtung der anatomischen Verhältnisse ihren Grund hat, vermag ich nicht zu sagen. Jedenfalls sind in der Literatur nur ganz vereinzelte Angaben in dieser Hinsicht zu finden. Um so bemerkenswerter erscheint die Angabe Drasches (11) über den Einfluss einer längerdauernden Kompression auf die tuberkulöse Lunge. Auf Grund eines grossen Sektionsmaterials hatte Drasche den Eindruck gewonnen, dass es unter dem Einfluss des Pneumothorax zu einer Hemmung des tuberkulösen Prozesses komme, während frischere Prozesse, namentlich frische Kavernen nur ganz selten beobachtet werden könnten. Diesen Beobachtungen Drasches reiht sich nun der Fall Forlaninis an, bei welchem es im Anschluss an einen mehrere Jahre bestehenden künstlichen Pneumothorax zu einer vollkommenen schwieligen Umwandlung der komprimierten Lunge gekommen ist.

Es dürfte daher von einigem Interesse sein, über die Untersuchungsresultate an einigen Fällen von künstlichem Pneumothorax zu berichten, um so mehr, als zurzeit die Frage über den Wert des therapeutischen Pneumothorax wieder brennend geworden ist.

Das Material, das mir für meine Untersuchungen zur Verfügung stand, hat mir Herr Prof. Brauer, auf dessen Anregung ich die nachfolgenden Untersuchungen rorgenommen habe, in liebenswürdigster Weise überlassen. Es sind im ganzen drei Fälle, von denen einer im hiesigen Institut zur Autopsie kam, während uns die beiden anderen von auswärts zugesandt wurden.

Die Lungen wurden im ganzen in Formalin gehärtet und Stücke aus verschiedenen Abschnitten des Lungengewebes in Paraffin eingebettet. Zur Färbung wurde neben einfacher Hämatoxylin-Eosinfärbung vorwiegend die Bindegewebsfärbung nach van Gieson, sowie die Elastikafärbung nach Weigert angewandt.

Wenn auch die kleine Anzahl von Fällen heute ein abschliessendes Urteil nicht zulässt, so scheint mir ihre Veröffentlichung doch von dem Gesichtspunkte aus gerechtfertigt, dass das ohnehin spär- 
liche Material einerseits eine Bereicherung erfährt und eventuell auch anderwärts die Aufmerksamkeit in erhöhtem Masse auf diesen Gegenstand gelenkt wird.

\section{Fall I.}

Frau O. L., 24 Jahre.

Anamnese: Seit der Kindheit blutarm und nervös. Im Januar 1906 mit Husten und Auswurf erkrankt. Fieber, Hämoptoë, Nachtschweisse, Gewichtsabnahme. Am 22. V. 1906 in das Sanatorium Dr. Turban, Davos-Platz aufgenommen.

Bei der Aufnahme besteht folgender Befund: R.V. Dämpfung bis zur dritten Rippe; R.H. bis zur Skapula; abgeschwächtes, bezw. vesikobronchiales Atmen. Auf der ganzen rechten Seite bronchiales Brummen und Stöhnen. Links über der Klavikula wenig gedämpft, mit rauhem Inspirium und verlängertem Exspirium. - Temperatur febril. Sputum: Tuberkelbazillen positiv. Mischinfektion mit Staphylococcus albus. Während des Aufenthaltes im Sanatorium ständig Fieber um 38,0 $0^{\circ}$ Appetitlosigkeit, Erbrechen, hochgradige Nervosität.

3. VII. 1906. Perkussion unverändert. R. über der Klavikula und Spina einige mittelblasige Rhonchi nach Husten, sonst durchweg vorn und hinten Stöhnen und Brummen, dazwischen da und dort zerstreut scharfes mittelblasiges Knacken.

10. VII. 1906. Wegen quälenden Hustens und starker nervöser Beschwerden Aufnahme in das tiefer gelegene Sanatorium Ebersteinsburg bei Baden (Dr. Rumpf). Von dort wegen rasch fortschreitender Verschlechterung zur Pneumothoraxtherapie an Brauer gesandt.

12. IV. 1907. Aufnahme in Marburg.

Sehr magere elende Patientin, psychisch sehr erregt, klagt fortwährend. Gewicht $41,9 \mathrm{~kg}$. Leichte Stomatitis catarrhalis. Schwellung und Rötung der Epiglottis. Dauernde Beschwerden beim Essen und Schlucken. Starke Nachtschweisse besonders in den Morgenstunden. Hautfarbe blass, rote Wangen; keine nennenswerte Zyanose. Ziemlich starke gleichmässige Kyphose der Brustwirbelsäule. Früher angeblich vollkommen gerade. Langer, schmaler und flacher Thorax; eingesunkene Interkostalräume; spitze Rippenwinkel. Claviculae springen stark vor; Schulterblätter stehen ab. Supra- und Infraklavikulargruben stark eingesunken, besonders rechts. Rechte Seite bleibt bei der Atmung stark zurück, Atmung sehr beschleunigt und rertieft. Bei psychischer Ruhe der Patientin wird die Atmung ebenfalls ruhiger. Die ganze rechte Seite vorne und hinten gleichmässig gedämpft; Wintrich oder Gerhardt nirgends vorhanden. Rechts überall lauteres oder leiseres Bronchialatmen mit reichlich klingenden Rasselgeräuschen 
jeder Grösse. Auch über dem oberen Lappen grossblasiges Rasseln. Die linke Spitze ist ganz wenig verkürzt, Atmung etwas verschärft. Bei wiederholter Untersuchung ist dort gelegentlich ganz vereinzeltes Knacken zu hören. Im übrigen ist der Schall links normal, ebenso das Atmungsgeräusch.

Herz etwas nach rechts verzogen, Herztöne rein, kräftig. Aktion erheblich beschleunigt, ca. 120. Puls auffallend gut gefüllt, von normaler Spannung.

Leichter Meteorismus; kein Milztumor. Keine Ödeme.

Auswurf sehr reichlich, klumpig, eitrig. Patientin hustet sehr viel. Narkotika wirken nur sehr wenig. Schlaf sehr leicht. Temperatur hoch fieberhaft.

Bei Durchleuchtung: Rechte Seite völlig dunkel, linke völlig hell. Links Zwerchfell gut beweglich, rechts nicht abzugrenzen.

Nächte andauernd schlecht. Patientin klagt über stechende Schmerzen unter der Klavikula. Dort Pleurareiben zu hören.

24. IV. 07. Operation. 0,02 Morph. Lokalanästhesie mit NovocainAdrenalin. Inzision. Man gelangt ohne Schwierigkeit in den Pleuraspalt, Druck $-5 \mathrm{~mm}$. $750 \mathrm{ccm} \mathrm{N}$ eingeblasen, Druck $+5 \mathrm{~mm}$.

Patientin ist nach der Operation sehr erschöpft. Atmung 60. Puls 140. Temperatur 37,3. Klagt über starkes Oppressionsgefühl. Abends schon wieder erheblich besser. - Die Temperatur steigt am nächsten Tage auf 39,0, bleibt aber dann im Durchschnitt erheblich niedriger als vor der Operation. - Hustenreiz dauernd stark. Auswurf etwas grösser als in den Tagen vor der Operation. Puls und Atmung deutlich langsamer.

26. IV. Klinisch sind alle Zeichen des Pneumothorax vorhanden. Das Atemgeräusch ist überall, auch hinten stark abgeschwächt. Die Rasselgeräusche sind noch in der Hilusgegend und weiter unten deutlich zu hören. Man hat den Eindruck, dass der Mittel- und Unterlappen fast allseitig von einem Luftmantel umgeben ist und dass auch der Oberlappen trotz breiter Adhäsionen teilweise von der Brustwand abgedrängt ist.

29. IV. Pneumothorax ein wenig kleiner, sonst unverändert.

30. IV. Zweite N-Einblasung. Anfangsdruck $-3 \mathrm{~mm}$. N-Menge $1250 \mathrm{ccm}$. Enddruck $+3 \mathrm{~mm}$. - Patientin hat zunächst nach der Einblasung wieder starke Beschwerden. Oppressionsgefühl. Schmerzen in der Lunge. Magendruck; kann nichts zu sich nehmen. Puls, Atmung und Temperatur sind gestiegen.

30. IV. Alle bedrohlichen Erscheinungen sind wieder zurückgegangen. 
1. V. Durchleuchtung: Pneumothorax grösser geworden. Verwachsungen zum Teil gedehnt, bestehen aber noch.

Temperaturen weiter abgesunken. Allgemeinzustand wesentlich gebessert. Puls und Atmung langsamer. Auswurf und Husten unverändert. Pleuraschmerzen sind besser geworden.

Die Stomatitis aphthosa ist fast vollkommen geschwunden.

14. V. Temperaturen wieder höher, jedoch tiefer als vor der Operation. Subjektives Befinden gut. Patientin nimmt an Gewicht etwas zu. - Pneumothorax hat sich nur wenig verkleinert. Schlaf ist wesentlich besser. Lästige Nachtschweisse bestehen noch.

15. V. Dritte Einblasung. Anfangsdruck $-3 \mathrm{~mm}$. N-Menge $1000 \mathrm{ccm}$. Enddruck $+8 \mathrm{~mm}$. Nach der Operation Befinden subjektiv schlecht; starke Beschwerden, die aber nach einigen Stunden wieder schwinden.

18. V. Durchleuchtung: Grosser Pneumothorax; kleines pleuritisches Exsudat.

24. V. Befinden der letzten 8 Tage in Vergleich zu den Zeiten vor der Operation bedeutend gebessert. Temperatur zwischen 37,0 und 37,6. Die Nachtschweisse lassen nach; der Auswurf ist geringer, Puls und Atmung gut. Patientin fühlt sich subjektiv wohl.

30. V. Temperaturen etwas gestiegen, bei subjektiv günstigem Befinden.

31. V. Plötzlich sehr heftige Schmerzen in der Cökalgegend; starke Druckempfindlichkeit. Faustgrosser Tumor, mässiger allgemeiner Meteorismus.

4. VI. Auf entsprechende Behandlung Beschwerden wieder zurückgegangen.

8. VI. Temperaturen wieder andauernd hoch, bis 40,0. Auffallende Gewichtszunahme (Exsudat).

11. VI. Durchfälle.

17. VI. Durchleuchtung: Das Exsudat reicht im Stehen bis zur Höhe der Mammilla, ist völlig frei beweglich, schlägt Wellen. Pneumothorax sonst unverändert.

18. VI. Punktion des Exsudates. Es entleeren sich nur $50 \mathrm{ccm}$ seröses Exsudat. Das Exsudat ist mikroskopisch und kulturell steril. An die Punktion anschliessend N-Einblasung. Der Druck steigt schon bei $100 \mathrm{ccm}$ an, so dass damit abgebrochen wird.

11. VII. Der Zustand ist durch die letzte Punktion nicht gebessert worden.

13. VII. Nochmalige Punktion mit nachfolgender N.-Einblasung. Es ergeben sich die gleichen Verhältnisse wie bei der Punktion vorher. 
29. VII. Punktion mit Einblasung. Etwa $150 \mathrm{ccm}$ Exsudat werden entleert, etwa ebensoviel $\mathrm{N}$ eingeblasen. Der Druck steigt sehr rasch. Exsudat steril, aber nicht mehr so klar wie früher, enthält Leukozyten und Lymphozyten.

18. VIII. Springende Temperaturen; dauernde Gewichtsabnahme; Zunahme der Auswurfmengen. Allgemeinbefinden verschlechtert sich andauernd. Die linke Seite bleibt auffallend gut. Es sind nur ganz selten einzelne knackende Rhonchi auf der linken Seite zu hören. Ständige Durchfälle. Leibschmerzen. Magen reicht bis zur Symphyse. Apfelgrosser Cökaltumor. Zeitweise Brennen beim Wasserlassen. Starke Nachtschweisse.

Im Laufe des September tritt wieder stärkere Stomatitis auf. Dauernde Durchfälle. Im linken Oberlappen werden stärkere krankhafte Prozesse bemerkbar.

Reichliche Taberkelbazillen im Stuhl. Starke Leibschmerzen und Schluckbeschwerden. Abmagerung; minimale Nahrungsaufnahme. Zunehmender Kräfteverfall. Patientin verlässt seit Juli das Bett. nicht mehr. Auswurfmengen erheblich gesteigert.

15. X. Die Stomatis hat sehr zugenommen. Fast die ganze Mundhöhle ist von einer mehr oder minder zusammenhängenden Soorschicht bedeckt, bei deren Entfernung die wunde, oft blutende Schleimhaut zutage tritt.

$16 \mathrm{X}$. Dauernd hohes Fieber. Exsudat im Sitzen etwa bis zur Mammilla reichend. Wahrscheinlich zahlreiche Adhäsionen, die das Exsudat zum Teil durchsetzen. Der 'Tiefstand der Leber spricht für tiefen Stand des Zwerchfells. - Herz noch immer auffallend kräftig. Linke Lunge ist anscheinend nur geringgradig an der Spitze affiziert. - Es wird nach reiflichen Überlegungen, an denen sich auch Dr. Brecke-Davos beteiligt, beschlossen, das Exsudat nach Rippenresektion zu entleeren und bei dieser Gelegenheit den Versuch zu machen, soweit wie möglich einzelne Verwachsungen zu lösen, um auf diese Weise einen ausgiebigeren Lungenkollaps zu erzielen. Es. ist selbstverständlich, dass in dem vorliegeuden Falle an eine extrapleurale Thorakoplastik, wie ich sie für andere Patienten empfahl, nicht zu denken war. Die Operation wird ausgeführt von Herrn Geheimrat Friedrich am

17. X. 1907. Inzision im 7. Interkostalraum, Eröffnung der Pleura. Resektion eines $8 \mathrm{~cm}$ langen Stückes der 7. Rippe. Es entleert sich trüb-seröses Exsudat. Rechter Unterlappen und Brustwand sind an der Hinterfläche verwachsen; die Verwachsungen sind leicht zu lösen. Beim Versuch, die festen Verwachsungen zwischen Unterlappen und Zwerchfell zu lösen, reisst eine grosse Kaverne ein, aus der reich- 
lich Eiter strömt. Die Verwachsungen werden vollkommen gelöst. Die Wunde wird tamponiert und drainiert.

19. X. Patientin fühlt sich nach der Operation sehr elend, nimmt nur flüssige Nahrung. Die Temperaturen sind auffallend gering. Puls schneller als sonst, aber kräftig.

21. X. Temperatur wieder angestiegen. Patientin fühlt sich subjektiv wieder besser.

25. X. Aus der Wunde fliessen ständig enorme Mengen Eiter ab; der Eiter riecht faulig und ist sehr schleimreich. Er enthält kleine weisse Klümpchen von Fettsäurenadeln. Tuberkelbazillen +; Kokken der verschiedensten Art. Kein Søor. Temperaturen ständig hoch (bis 39,0). Puls auffallend gut. Spärliche Durchfälle; häufig Tenesmen.

Beginnender Dekubitus am Kreuzbein. Sehr quälenden Husten. Viel Nachtschweisse. Nahrungsaufnahme besser als vor der Operation.

31. X. Zustand unverändert. Puls immer noch gut. Starke stechende Schmerzen links neben dem Herzen. Von der linken Axilla bis zum Herzen ein grobes Reiben hörbar, sonst ist die linke Seite unverändert.

3. XI. Schmerzen und Reiben völlig geschwunden. Beginnender Dekubitus über den Trochanteren.

6. XI. Mittags verhältnismässig gutes Befinden. Abends plötzlich ein Anfall schwerer Zyanose mit Kollapserscheinungen. Sauerstoffinhalationen; Kampfer. Nach 11/2 Stunden wird die Patientin. wieder munter.

7. XI. Morgens leidliches Befinden; Patientin ist sehr matt. Puls weniger gut als bisher. Mittags neuer Anfall von Zyanose, der sich wieder beseitigen lässt.

$6 \mathrm{Uhr}$ abends neuer, schwerer Anfall von Zyanose und Kollaps. Zunehmende Herzschwäche. Trachealrasseln.

8 Uhr p. m. Exitus letalis.

Sektionsprotokoll (Dr. Gra etz). Kleine, hochgradig abgemagerte Leiche mit blasser Hautfarbe. Durch die Haut hindurch ist die Konfiguration des Skeletts deutlich zu erkennen. Die Muskulatur ist vollkommen atrophisch, das Unterhautfettgewebe fast völlig geschwunden. Totenstarre besteht nicht. An den abhängigen Partien geringe blasse Totenflecke. Über den beiden Trochanteren, desgleichen über dem Kreuzbein bestehen grosse Dekubitalgeschwüre. In der rechten Thoraxseite befindet sich in der Axillarlinie im 7. Interkostalraum eine ca. $12 \mathrm{~cm}$ lange, parallel zu den Rippen verlaufende, breit klaffende Inzisionswunde, die mit Gaze tamponiert ist. Die VII. Rippe ist reseziert, ihre Enden ragen aus der Wunde heraus. Die Wunde, 
deren Ränder mit schmierigem Eiter belegt sind, führt in die offene Pleurahöhle. Die Wände der Pleurahöhle sind ebenfalls mit dickem, schmierigen Eiter reichlich belegt. Die rechte Lunge ist völlig kollabiert und als grosser, fester Knollen an der Wirbelsäule zu fühlen. An der Spitze und an der der Basis entsprechenden Seite der kollabierten Lunge fühlt man strangförmige Verwachsungen mit der Umgebung, die sich ohne grosse Schwierigkeiten lösen lassen. Im übrigen ist die komprimierte Lunge frei im Pleuraraum beweglich. - Die linke Lunge ist in ganzer Ausdehnung teils strangförmig, teils flächenhaft mit der Pleura verwachsen und lässt sich nur mit grosser Mühe auslösen. Die Pleurahöhle ist frei von Flüssigkeit. - Herzbeutel normal. Das Herz enthält ein durch die Kontraktion des Ventrikels schlingenförmig gestaltetes Speckgerinnsel. Muskulatur in allen Teilen etwas șchlaff, rechts nicht hypertrophisch; links dentlich atrophisch. Klappen normal.

Die rechte Lunge ist hochgradig zusammengezogen, derb deformiert. Die Oberfläche ist überall von trockenen Fibrinlagen bedeckt, welche von der Lunge her organisiert werden. Spitze fibrinös adhärent. Über dem Oberlappen besteht eine frische, dünne, fibröse Schwiele zwischen dem Fibrin und der Pleura. Der Unterlappen ist nur wenig schwielig verändert. Im Oberlappen finden sich vier Fistelgänge, der grösste von Bleistiftdicke, welcher durch das Schwielengewebe hindurch eine Kommunikation zwischen kleinen und grösseren Kavernen und dem Lungenraum herstellen. Die Kavernen sind zusammengefaltet, fast leer. Das Lungengewebe enthält ziemlich reichliche, trockene, käsig pneumonische Herde in völlig atelektatischer Umgebung. Keine frische Tuberkelaussaat. Ein erbsengrosser derartiger Herd wird gerade im Durchbruch durch die Pleura gefunden.

Im Unterlappen zeigt sich auf der grossen Kante, der Spitze des Unterlappens entsprechend, eine $2 \mathrm{~cm}$ lange und $4 \mathrm{~cm}$ breite Öffnung, welche in eine $3 \mathrm{~cm}$ tiefe Kaverne führt. Diese Kaverne kommuniziert mit dem obersten Ast des Unterlappenbronchus. Sie zeigt abgerundete Ränder und namentlich vollkommene Abrundung der Übergangsstelle zwischen Kaverne und Pleura. Ein ähnlicher grosser, abgeglätteter Kavernenraum liegt an der hinteren Fläche der Lunge; auch an ihm lassen sich Kommunikationen mit grösseren Bronchialästen feststellen. Ferner ist der untere Abschnitt des Lungenlappens von einer grösseren Anzahl mehr oder weniger tiefer Gruben wie angefressen. Jede dieser Gruben entspricht einer Verkäsung eines scharf begrenzten, daräber liegenden Teiles, und es lässt sich erweisen, dass es sich dabei um peribronchitische Verkäsungen han- 
delt, welche die kleinen, zylindrisch erweiterten Bronchien bis zur Pleura umgeben. Die grösseren Bronchien sind intensiv gerötet und eng bis zum Abgang von der Bifurkation. Die Schleimhaut erscheint schwer entzündlich, aber nicht sicher tuberkulös. Die Bronchien haben grösstenteils das Aussehen alter Fistelgänge, zum Teil sind sie in ihren peripheren Enden zylindrisch erweitert, oder gehen direkt in kleine kugelige Kavernen über.

Die linke Lunge ist etwas vergrössert und gebläht. Sie enthält eine grosse Anzahl käsig pneumonischer, meist ganz frischer Herde, ausserdem im Oberlappen eine ganz frische mit reichlichem, tuberkulösem Brei, tuberkulösen Linsen und grösseren Nekrosefetzen vollkommen angefüllte Kaverne, von platter, länglicher Beschaffenheit, deren Verlauf deutlich dem Hauptbronchus des Oberlappens entspricht.

Die Herde des Unterlappens sind unverkennbar sehr frisch und annähernd gleich alt. Nirgends besteht eine Aussaat miliarer Tuberkeln. Bronchien im allgemeinen normal, nur wenig entzündet. Bronchiallymphdrüsen vergrössert, mit Tuberkeln durchsetzt. Nirgends findet sich eine Pleuraperforation. Doch liegen zwei käsige Herde dicht unter der Pleura und sind unmittelbar dem Durchbruch nahe. Mikroskopische Untersuchung (Stück aus dem Unterlappen).

Die Lunge ist im allgemeinen atrophisch; die Alveolarwände meist sehr schmal. Die Alveolen wenig oder gar nicht erweitert; die meisten enthalten einige abgestossene Epithelien und Leukozyten. Streckenweise finden sich Alveolen, die ödematöse Infiltrate, sowie Fibrinexsudat einschliessen. Die Fibrinmassen sind häufig sehr derb, glasig, kanalisiert und enthalten weitere oder engere, prall mit Blut gefüllte, dünnwandige, neugebildete Gefässe, sowie allerlei sonstige junge organisierende Gewebselemente. In derartigen Alveolen zeigen sich die Epithelien meist in starker Vermehrung, so dass sie in Gestalt kubischer, dicht gedrängter Säume das Lumen auskleiden. Weiterhin finden sich in der Umgebung der grösseren Blutgefässstämme und Bronchien deutlich vermehrte breite Bindegewebszüge. Neben denselben zeigen die Alveolen stärkere Epithelregeneration. Beide Erscheinungen zusammen entsprechen dem Bilde älterer Lungennarben. Die Ausbreitung der Narben weist darauf hin, dass chronische Perivaskulitis und Peribronchitis bestanden hat, zur Zeit der Untersuchung aber in den Heilungszustand übergegangen war. In unmittelbarer Beziehung zu diesen Narbenbildungen, bisweilen als deutliche Zentra junger Narben findet sich eine Anzahl von isolierten Tuberkeln mit nekrotischem Zentrum und schmaler Epitheloidzellenzone. Der Rand solcher käsiger Herde zeigt ausgeprägte fibröse Wucherungen, zwischen denen hier und da Langhans- 
sche Riesenzellen und kleinste Tuberkelknötchen vorkommen. Die Schwielen um die Knötchen berum greifen zuweilen ziemlich tief in die umgebenden Alveolarwände hinein. Das ganze Bild entspricht. einer fibrösen Vernarbung und Abkapselung von Tuberkeln, von denen offenbar längere Zeit vor dem Zeitpunkt der Untersuchung ein umfangreicher Reizzustand auf die benachbarten Teile ausgegangen war.

Ein anderer, der Lungenoberfläche nahegelegener Schnitt von grösserer Ausdehnung zeigt gleichfalls ziemlich reichlich die perivaskulären älteren Schwielenbildungen, sowie im allgemeinen eine ausgeprägte Atelektase. Manche Gebiete erscheinen sehr stark hyperämisch, namentlich auch die Umgebung der hier zahlreich vorhandenen Tuberkelknötchen. Diese selbst stellen im Gegensatz zu dem. zuerst beschriebenen Präparat, vielfach frischere Verkäsungsstadien dar; sie zeigen viel reichlichere Epitheloidkerne, sehr grosse Quantitäten zerfallener, aber auch noch nicht unfärbbar gewordene Kerne und einen sehr zellreichen, überall mit nektorisierender Pneumonie der anstossenden Alveolen kombinierten Entzündungssaum. Bindegewebswucherungen im Sinne der fibrösen Ausheilung fehlen auch hier nicht, sind aber im ganzen spärlich. Frische käsige Herde von grosser Ausdehnung erreichen die Oberfläche der Lunge und haben dieselbe durchbrochen. Man gewinnt nach dem Gesamtbild den Eindruck, dass dieser Durchbruch relativ dicht vor dem Untersuchungstermin erfolgt sein könnte. Hiernach würden die peripheren $A b-$ schnitte des Lungengewebes eine raschere Entwickelung dichtgestellter, käsiger Herde aufweisen, die zur selbständigen Perforation vom Lungengewebe her gegen und durch die Pleura führen, während die zentral gelegenen Abschnitte der Lungen mehr narbige Prozesse aufweisen.

Die andere Lunge zeigt keine Andeutung von Schwielenbildung im Lungengewebe, dagegen enthalten ihre Bronchien bisweilen dicke, fibrinöse Ausgüsse mit zerfallenen Kernen und die Abveolen sind in weitem Umfange durch Oedem, katarrhalische Exsudate, Fibrinmassen oder käsig pneumonisches, nekrotisches Material angefüllt. Überall zeigen die Übergangsstadien, dass der Prozess der Verkäsung sich fortwährend an anderen Stellen aus katarrhalischer Pneumonie neu entwickelt.

Das Gesamtbild deutet auf eine rasche und ausgedehnte Entwickelung der käsigen Pneumonie. An einer Stelle finden sich auch käsige Lungenvenenthromben.

\section{Fall II.}

St. Theophil. 20 Jahre.

Anamnese: Vater phthisisch seit 30 Jahren, lebt, arbeitet; Nutter gesund. Übrige Geschwister gesund. Patient erkrankte mit. 
6 Jahren an Pertussis; während der Schulzeit Masern, Diphtherie, bäufig Angina. Frühjahr 1904 Husten mit wenig Auswurf, kleine Hämoptoe. Husten verschwand wieder, kam im Juni 1904 wieder mit vermehrtem Sputum und Fieber $\left(-39^{\circ}\right)$. Fieber verschwand wieder langsam, aber während der ganzen Zeit bis zur Anlegung des Pneumothorax häufig Lungenblutungen mit häufigen Fieberschüben. Abmagerung seit $1^{1 / 2}$ Jahren. Nachtschweisse vorübergehend.

Status der Baseler Heilstätte (Dr. Nienhaus):

29. VIII. 1907. Ernährungszustand unter Mittel. Thorax flach, untere Partien eingesunken. Leichte Skoliose der unteren Brustwirbelsäule, konvex nach links. Körpergewicht $61 \mathrm{~kg}$. Herz: O. B.

Perkussion: R.V. über der Klavikula leicht relativ gedämpft, abnehmend bis zur IV. Rippe. L.V. über der Klavikula leicht gedämpft, im I. Interkostal-Raum ganz leicht; unten aussen Schall verkürzt. R.H. leicht relativ gedämpft, abnehmend bis zum Angulus scapulae. L.H. über der Spina relativ gedämpft, abwärts bis unter die Spina abnehmend.

Auskultation: R.V. s. sp. abgeschwächtes unbestimmtes Atmen, ähnlich bis zur IV. Rippe; im IV. I.-R. neben dem Sterum leises vesikuläres-bronchiales Inspirium und Exspirium; unterhalb Atmung anrein vesikulär, oberhalb bis zur IV. Rippe feinblasige feuchte Rhonchi in mittlerer Zahl nachHusten. R.H. s.sp.Atmung abgeschwächt, unbestimmt, von der Spina abwärts unreines vesikuläres bis bronchiales Inspirium mit verlängertem Exspirium bis zum Angulus, unterhalb vesikuläres bis bronchiales Atmen. L.V.s.cl. unrein vesikuläres Inspirium, verlängertes Exspirium bis II. Rippe. Im II. I.-R. verschärftes Inspirium, neben der Herzdämpfung vesikuläres bis bronchiales Inspirium mit verlängertem Exspirium. L.H. s. sp. unreines Inspirium mit verlängertem Exspirium; infra unreines vesikuläres Atmen.

Larynx: Stimmbänder diffus gerötet; an der hinteren Kommissur beiderseits der Mittellinie eine halbkugelige Vorwölbung, Schleimhaut intakt.

Während der Kur häufig wegen Fieber und Hämoptoe bettlägerig, gegen Schluss der Kur 2 Monate ausser Bett. Gewichtsabnahme 5-6 kg. Nach Austritt aus der Heilstätte war Patient bis September 1907 in einer Pension in Davos-Dorf. Er war sehr häufig bettlägerig, machte verschiedene grössere Blutungen durch und hatte auch Fieber. - Am 9. September Wiederaufnahme in die Heilstätte.

Status (Dr. Ni enhaus): 10. IX. 07. Herzgrenzen oben mit Rand der III. Rippe, links 2 Querfinger neben dem Sternum, rechts 2 Querfinger neben dem Sternum. - Lunge: R. V. untere Grenze mit Rand der VI. Rippe, verschieblich, links wie rechts; H. Processus spin. dors. X. 
R.V.s.cl. relative Dämpfung, abnehmend bis zur IV. Rippe, von da ab nach unten zunehmende Dämpfung. - L.V. s. cl. leicht relative Dämpfung auch im I. I.R.

R.H. s. sp. relative Dämpfung, langsam abnehmend bis unten. L.H. s. sp. relative Dämpfung weniger wie rechts, abnehmend bis gegen die Mitte der Skapula.

Auskultation: R.V.s.cl. Atmung durch Rhonchi verdeckt, mit lang hauchendem bronchialem bis vesikulärem Exspirium; unterhalb allgemein von vesikulärem bis bronchialem Charakter, grossenteils durch Rhonchi verdeckt. Von oben bis unten zahlreiche feuchte, bis mittelblasige, klingende und halbklingende Rhonchi. R.H. Atmung vollständig verdeckt durch zahlreiche mittel- bis grossblasige klingende und halbklingende Rhonchi. - L.V. s. cl. verschärftes vesikular-bronchiales Atmen, Exspirium etwas verlängert, unterhalb ähnlich. An der unteren Grenze in der Mammillarlinie ein feines inspiratorisches Knacken. L.H.s. sp. vesikuläres-bronchiales Inspirium, leise hauchendes, verlängertes Exspirium; unterhalb etwas verschärftes vesikuläres Atmen bis bronchiales Inspirium, rauhes, leise knurrendes Exspirium bis unten. Vom Angulus bis unten ziemlich zahlreiche, feinblasige inspiratorische Rhonchi. Wegen des äusserst ungünstigen Verlaufes, vor allen Dingen aber wegen der häufig sich wiederholenden Blutungen und nachmaligen Fieberschübe drängt Patient und dessen Familie anf Anlegung eines künstlichen Pneumothorax, trotzdem ihm und der Familie die Gefahren eines solchen Eingriffes wegen des Befundes auf der linken Lunge ausdrücklich betont werden.

11. IX. 1907. Anlegung eines künstlichen Pneumothorax (Prof. Brauer). Im V.Interkostalraum Einfliessenlassen von $900 \mathrm{ccm}$ Stickstoff. Der Eingriff wird ohne Schmerz überstanden. Nachher ist sitzende Stellung notwendig, da beim Liegen Dyspnoe eintritt. Später etwas Hustenreiz und Schluckbeschwerden. Einmal Erbrechen.

12. IX. Keine besonderen Beschwerden, geringe Dyspnoe.

13. IX. Einblasen von $1000 \mathrm{ccm}$ Stickstoff, nachher leichte Dyspnoe und leichte Schluckbeschwerden.

16. IX. Vermehrtes Sputum. L.H.U. zahlreiche Rhonchi. Rechts ausgesprochener Pneumothorax.

21. IX. Tympanie rechts ist langsam zurückgegangen. $1200 \mathrm{ccm}$ Stickstoff werden gut ertragen.

4. X. Keine Änderung, relatives Wohlbefinden $900 \mathrm{ccm}$. Niedrige Temperatur - 37,6.

5. X. Vorübergehend etwas Atemnot, Appetit und Schlaf mässig. Zwerchfell R.H.U. beim II. Lendenwirbel, 3 Querfinger tiefer wie links. 19. X. In der Nacht vom 17. bis 18. X. Schüttelfrost. Temperatur 38,9. 
Schmerzen R.U. seitlich. Vermehrte Dyspnoe. R.H.U. 2 Querfinger hohe Dämpfung. (Linke Lunge steht soviel tiefer.) R.V. keine Dämpfung. R.V. und seitlich von der V. bis VII. und IX. Rippe Haut hyperästhetisch. Auf Pyramidon Schlaf.

20. X. Morgens starke Kyanose, Dyspnoe. Temperatur 38,5.

21. X. Dyspnoe geringer. R.H.U. schmälere Dämpfung. Hyperästhesie verschwunden. L.H. von der Mitte bis unten zahlreiche feuchte, mittel- und grossblasige Rhonchi.

22. und 23. X. Status idem. Temperatur $38,5^{\circ}$.

24. X. Dyspnoe vermehrt. Sanerstoffinhalation. Temperatur 37,20.

30. X. Status idem. Morgens Cyanose; Cheyne-Stokes; auf Sauerstoffinhalation und Oleum camphorat subkutan, Besserung. Temperatur $37,5^{0}$.

2. XI. Unter zunehmender Dyspnoe allmählich zunehmende Schwäche; stets Sauerstoffinhalationen. Von $5^{\text {b }}$ a. m. bewustlos, $7^{\text {h }}$ 5. a. m. Exitus letalis.

\section{Sektionsprotokoll.}

(Im Auszug; nach dem Sektionsberichte aus Davos (Dr. Nienhaus) und dem eingesandten Präparat. Dr. Graetz).

Bei Eröffnung der Pleurahöhle unter Wasser entweichen Gasblasen. Im rechten Pleuraraum gut 21 eitriger, an der Oberfläche grünlich schimmernder Flüssigkeit. Die rechte Lunge ist stark komprimiert, fest gegen die Wirbelsäule gedrückt. Die Basis ist fest mit dem Zwerchfell verwachsen. Von der Spitze ziehen 3 bandförmige Narbenstränge zur Brustwand. Die linke Lunge ist durch leicht lösliche frische Verklebungen mit der Pleura costalis verbunden, die Spitze frei beweglich.

Das Herz liegt an normaler Stelle und lässt nichts Abnormes erkennen.

Die rechte Lunge ist zirka zweifaustgross, vollkommen atelektatisch, von derber, fleischartiger Konsistenz. Die Pleura ist über allen Lungenbezirken stark schwielig verdickt; an der Spitze des Oberlappens finden sich 3 bandförmige, schwielige Adhäsionen. Auch zwischen den einzelnen Lungenlappen bestehen schwielige Adhäsionen, so dass die Grenze der einzelnen Lappen nur schwer festzustellen ist. Im Oberlappen liegt eine zirka $7 \mathrm{~cm}$ lange, mit Eiter gefüllte Kaverne, deren Ränder dicht aneinander gepresst sind. Das benachbarte, komprimierte Lungengewebe, das etwa Mandarinengrösse zeigt, ist von zahlreichen, gerstenkorngrossen Tuberkeln durchsetzt und lässt eine muskeläbnliche Konsistenz erkennen. Eine weitere, etwa haselnussgrosse, eitergefüllte Kaverne liegt inmitten komprimierten Lungenge- 
webes, in den hinteren Abschnitten des Oberlappens. - Das Gewebe des Mittellappens weist eine gleiche Beschaffenheit auf, wie das des Oberlappens. Die grossen Bronchialstämme klaffen breit und besitzen eine auffallend starke, starre Wand. - Der Unterlappen ist von zahlreichen, bis erbsengrossen, im Zentrum verkästen Tuberkelherden durchsetzt, die das Zentrum derber Bindegewebsschwielen bilden. Die unters tenPartien des Unterlappens zeigen pneumonische Infiltration. Auch hier zeigen die grossen Bronchialstämme breit klaffende Lumina und starre, derbe Wandungen. In sämtlichen Abschnitten der Lunge fällt die starke Blässe und Pigmentarmut des Lungengewebes auf.

Linke Lunge sehr voluminös, ihre Pleura leicht getrübt. In der Spitze des Oberlappens eine zehnpfennigstückgrosse, schiefrig gefärbte flache Narbe. Das Gewebe des Oberlappens ist ziemlich ödematös, lufthaltig. In der Mitte des Lappens, sowie an seinem unteren Rande mehrere bis pfefferkorngrosse tuberkulöse Herde mit teils eitrigem, teils käsigem und verkalktem Inhalt. Auf der Hinterfläche des Unterlappens bestehen mehrere schiefergraue, narbige Einziehungen. Auf dem Durchschnitt sieht man neben mehreren bis haselnussgrossen älteren Käseherden zahlreiche submiliare, leicht opake Tuberkelknötchen.

Die Sektion der übrigen Organe ergab nichts Besonderes.

Mikroskopische Untersuchung.

(Stück aus dem Unterlappen der rechten Lunge, aus der Gegend des vorderen Randes.)

Das mikroskopische Strukturbild der Lunge erinnert an die Bilder, wie ich sie bereits im Falle I beschrieben habe; doch erscheint die Bindegewebsentwicklung in diesem Lungenabschnitt schon bei Lupenvergrösserung auffallend viel stärker. Das Lungengewebe erscheint zum Teil vollkommen schwielig umgewandelt, nur an vereinzelten Stellen lassen hochgradig komprimierte und atrophische Alveolen die ehemalige Gewebsstruktur erkennen. Das ganze komprimierte Gewebe des Unterlappens ist von einer mehrere Millimeter breiten Bindegewebsschwiele umgeben. Auf der Oberfläche der Schwiele erkennt man sehr zellreiche, stellenweise nekrotische Auflagerungen, wahrscheinlich tuberkulöser Natur. Die Auflagerungen sind von einem fein fibrillären Bindgewebe durchzogen, das in direkter Beziehung zur darunterliegenden Pleuraschwiele steht. Auch nach dem komprimierten Lungengewebe $\mathrm{zu}$ verlaufen von der Pleuraschwiele aus teils feinere, teils derbere Stränge eines fibrillären Bindegewebes, so dass eine scharfe Grenze zwischen Pleuraschwielen und Lungengewebe nicht zu erkennen ist. Bei der Weigertschen Elastikafärbung wird 
diese Grenze durch eine schmale, gewellt verlaufende Linie von elastischen Fasern markiert. Das atrophische und komprimierte Lungengewebe ist von einer grösseren Anzahl, im Zentrum verkäster Tuberkelherde durchsetzt, die ringsum von einem dichten Wall frischeren und älteren Bindegewebes umgeben sind, welches sie nach Art einer Kapsel vollkommen einscheidet. Die Verkäsung der Zentra ist eine ausgesprochen trockene. Nach dem verkästen Zentrum der Knötchen zu ziehen von der bindegewebigen Kapsel aus feine Stränge fibrillären Bindegewebes, welche die verkästen Massen von allen Seiten durchziehen. In der Umgebung der grossen Gefässe und Bronchien ist es ebenfalls zur Entwickelung grosser, derber Bindegewebsschwielen gekommen, welche wohl analog den Verhältnissen im ersten Falle, als der Ausdruck einer ausgeheilten Perivaskulitis und Peribronchitis anzusehen sind. Die perivaskulären und peribronchialen Schwielen stehen durch Stränge von Bindegewebe mit den fibrösen Kapseln der verkästen Tuberkelherde in Verbindung, so dass das Lungengewebe nach allen Seiten hin von Bindegewebsnarben durchzogen ist. - Neben den einzelnen tuberkulösen Herden finden sich auch Stellen im Lungengewebe, die eine pneumonische Infiltration aufweisen. Aber auch in diesen Gebieten ist eine deutliche Organisation durch junges Bindegewebe nachweisbar. - Neben diesen Heilungsvorgängen finden sich aber auch unzweifelhaft frische tuberkulöse Herde. Doch lässt die immerhin spärliche Anzahl solcher frischer Tuberkel auf eine nur geringe Tendenz des Prozesses zum Fortschreiten schliessen. Die frischen Tuberkelknötchen liegen vorwiegend in der Umgebung der abgekapselten Käseherde. Tuberkelbazillen sind sowohl in den älteren wie in den frischeren Herden nur in ganz spärlichen Mengen nachzuweisen. Das elastische Gewebe der Lunge ist an den Stellen der Schwielenbildung vollkommen geschwunden, nur in der Umgebung der grösseren Gefässstämme ist es in geringen Resten erhalten. - Auffallend ist auch mikroskopisch der fast vollkommene Pigmentmangel, namentlich auch innerhalb der Narbenbildungen. Die Gefässe sind stark erweitert, zum Teil prall gefüllt.

Die Untersuchung des Lungengewebes aus dem Mittel- und Oberlappen, sowie aus mehr zentral gelegenen Teilen des Unterlappens ergibt mikroskopisch im ganzen die gleichen Bilder wie ich sie oben beschrieben habe. Auch hier finden sich fibröse Vernarbungen tuberkulöser Herde neben frischen Tuberkeleruptionen. Wenn man die Bindegewebsbildung in den einzelnen Lungenabschnitten miteinander vergleicht, so fällt sofort der graduelle Unterschied der Schwielenbildung auf; man kann feststellen, dass die Bindegewebsbildung um so stärker auftritt, je geringer das Volumen des komprimierten Lungen- 
abschnittes ist. Eine stärkere Ausbreitung der frischen Prozesse ist indessen auch in den Partien nicht nachzuweisen, in welchen die Schwielenbildung weniger ausgeprägt erscheint.

\section{Fall III.}

Walther R., 26 Jahre. (L. Spengler und Brauer.)

Anamnese: Ein Bruder des Patienten ist tuberkulös, sonst ist bezüglich der Tuberkulose eine Heredität nicht nachzuweisen. Patient hustet seit November 1901. Ende November eine kleine Blutung. Im Winter 1901/02 war Patient in Görbersdorf, wo er mehrmals Blutungen hatte. Den Sommer 1902 verbrachte er zu Hause und befand sich seit 7. XII. 02 mit geringer Unterbrechung in Davos. Temperatur bei der Aufnahme $38,4^{0}$; leichtes Fieber $\left(37,7^{\circ}\right)$ bis Spätsommer 1903. Auf Behandlung mit Tuberkulin im Herbst 1903 vollkommen fieberfrei, so dass er 1904 und 1905 Bureauarbeit ausführen konnte. Darnach trat eine Verschlechterung des Zustandes ein; am 1. VIII. 06 Lungenblutung; Mitte März 07 Pleuritis sicca rechts mit Temperatur bis $38,7^{\circ}$. Deutliche Gewichtsabnahme. Seit einigen Wochen ist Patient wieder fieberfrei, aber der Zustand der rechten Lunge hat sich seit einem Jahre bedeutend verschlechtert, Kavernenbildung im Oberlappen, auch sonst rasches Fortschreiten der Prozesse. Damit fast dauernd Fieber. 23. VIII. 07 Gewicht 114 Pfund, Puls 90-100, Temperatur $36,8-37,2^{\circ}$. Im Auswurf zahlreiche Tuberkelbazillen und elastische Fasern. Intensive Dämpfung über der ganzen rechten Lunge. R.V. oben über der Klavikula und im 1. Interkostalraum Atmung amphor. und zahlreiche klingende Rhonchi. Im 2. Interkostalraum Atmung broncho-vesikulär, ebenso im 3. Interkostalraum. Im 4. und 5. Interkostalraum rechts Atmung leise, rauh, Exspirium hauchend, klingende und halbklingende Rhonchi. R.H. oben über dem Oberlappen Atmung broncho-amphorisch mit klingenden Rhonchi. Über dem ganzen Unterlappen Atmung leise, rauh, Exspirium verlängert und zahlreiche Rhonchi, besonders mittlere und halbklingende.

Linke Lunge hinten und vorne Atmung verschärft, Exspirium verlängert, aber ohne Rhonchi. Befund der linken Lunge vom 2. V. 06 :

Links vorne über der Klavikula Inspirium scharf, Exspirium hauchend, aus der Tiefe bronchial; spärliches leises Knattern beim Husten. Im 1. Interkostalraum Inspirium scharf, Exspirium verlängert, Knattern nach Husten; im 2. Interkostalraum der gleiche Befund. Im 3.-5. Interkostalraum Inspirium rauh, Exspirium etwas 
verlängert; wenig trockenes Knattern nach Husten. Linker Oberlappen hinten wie vorne. Linker Unterlappen frei.

Patient war allmählich sehr elend geworden, er war sich des Ernstes der Situation voll bewusst und bat daher im Hinblick auf die Erfolge, die er bei anderen sah, um Anlegung eines künstlichen Pneumothorax. Die zweifelhafte Aussicht, die die Anlegung eines Pneumothorax bot, liess eine Zeitlang zögern; es wurde dann aber doch am 14. IX. 07 die Pneumothoraxtherapie als Ultimum refugium versucht. Es gelang zunächst einen kleinen Pneumothorax von $300 \mathrm{ccm}$ Stickstoff anzulegen und diesen bei späteren wiederholten Punktionen auch um Stickstoffwerte von $300-600 \mathrm{ccm}$ zu vermehren. Einige Male trat ein stärkeres Hautemphysem auf.

Trotz wiederholter Nachfüllung des Pneumothorax konnten aber die Verwachsungen nicht ganz gelöst werden. Der Unterlappen war in den hinteren Partien adhärent, war nur teilweise zu komprimieren. Es sank für eine Zeit das Fieber. Das Allgemeinbefinden blieb zunächst unverändert, wurde dann aber weiterhin schlechter. Es bildete sich am 12. X. 07 ein mässig grosses pleuritisches Exsudat. Am 28. X. 07 trat unter fcrtschreitendem Kräfteverfall der Tod ein.

Sektionsprotokoll. (Dr. Graetz. Nach dem Protokoll aus Davos und dem eingesandten Präparat.)

Mittelgross, schwächlich gebaut, schlecht genährt, Hautfarbe blass, an den abhängigen Partien geringe Totenflecke. Fettgewebe und Muskulatur spärlich entwickelt. Von der rechten vorderen Axillarlinie bis zum Sternum, vom linken Sternalrand bis zur linken Axilla, nach unten bis zur VII. Rippe leichtes Hautemphysem.

Aus der rechten Pleurahöhle entweichen Gasblasen. Die rechte Pleura bildet eine mehrkammerige, mit trüber, rotbrauner Flüssigkeit gefüllte Höhle. Die Pleura costalis ist mit einem dicken, schmierigen, zum Teil eitrigen Belag bedeckt, der stellenweise fest auf der Unterlage haftet. Die rechte Lunge ist von vorne und von der Seite her fest gegen die Wirbelsäule gepresst, nur in den hinteren Partien ist sie in ganzer Ausdehnung fest mit der Thoraxwand verwachsen; auch an der Basis des Unterlappens bestehen feste Verwachsungen mit dem Zwerchfell. Durch einige bandförmige Adhärenzen, welche nach der vorderen seitlichen Brustwand ziehen, wird der Pleuraraum in mehrere, miteinander verbundene Kammern geteilt. Die rechte Pleurahöhle ist nach links gegen das Mediastinum zu vorgebuchtet. - Die linke Lunge ist in ziemlicher Ausdehnung teils flächenhaft, teils strangförmig mässig fest mit der Brustwand verwachsen.

Im Herzbeutel vermehrte Flüssigkeit. Das Herz selbst ist 
schlaff, die Muskulatur transparent, sonst ohne Befund. Klappen intakt.

Die rechte Lunge lässt sich nur unter gleichzeitiger Auslösung eines Teiles der Pleura costalis herausnehmen. Die ganze Lunge ist hochgradig komprimiert, etwa kleinkindskopfgross. Die Oberfläche der einzelnen Lappen ist überall und zwar auch dort, wo Verwachsungen bestanden, von einer derben fibrösen Schwarte überzogen, auf deren Oberfläche stellenweise zablreiche Tuberkelknötchen verstreut. liegen, die zum Teil ein verkästes Zentrum erkennen lassen. Zwischen Mittel- und Unterlappen verlauft ein mehrere Millimeter dickes Septum, das mit schmierigem Belag bedeckt und von zablreichen kleinsten Tuberkelknötchen durchsetzt ist. Ober- und Mittellappen sind vollkommen atelektatisch, von derber, fleischartiger Konsistenz. In den Spitzenpartien des Oberlappens ist das atelektatische Gewebe von breiten, weissglänzenden Bindegewebsschwielen durchzogen. Hier im Oberlappen liegt eine zirka kirschgrosse eitergefüllte Kaverne mit teilweise abgeplatteten Rändern, die gegen die Umgebung durch eine grauweisse Bindegewebsschwiele abgekapselt ist. Die Lumina der grösseren und kleineren Gefässe und Bronchialstämme klaffen breit, ihre Wand ist starr und auffallend derb. In der Lmgebung der Bronchien und Gefässe ist das Lungengewebe vielfach von derben, eigentümlich glasigen, grauweisslichen Strängen durchzogen. Frische Tuberkelerruptionen oder verkäste Herde sind makroskopisch weder im Oberlappen, noch im Mittellappen zu erkennen. Auffallend ist auch hier namentlich stellenweise der Pigmentmangel der Schwielenbildungen.

Im Unterlappen findet sich eine taubeneigrosse Kaverne mit eitrigem Inhalt und unebener Wandung. In das Lumen der Kaverne springen kleine varikös erweiterte Gefässchen vor. Das Gewebe des Unterlappens enthält noch geringe Mengen Luft und zeigt nur mässig derbe Konsistenz. Bronchien und Gefässe zeigen hier nichts Auffälliges. Im Gewebe verstreut finden sich eine grössere Anzahl hirsekorn- bis linsengrosser Tuberkelknötchen hier und da mit verkästem Zentrum, teils einzeln, teils in Gruppen zusammenstehend. Das übrige Gewebe ist mehr pneumonisch infiltriert.

Die link e Lunge ist emphysematös gebläht, sehr voluminös. Nur über einem Teil des Oberlappens ist die Pleura spiegelnd, in den übrigen Partien ist sie trübe, mit feinen fibrinösen Auflagerungen bedeckt. Die Pleura des Unterlappens ist fast überall schwielig verdickt. Das Gewebe des Oberlappens erscheint rotgrau, stark ödematös, stellenweise gut lufthaltig. An der Spitze befinden sich zwei etwa bohnengrosse, schiefrig indurierte Herde, in der unteren Hälfte des 
Lappens eine walnussgrosse, eitergefüllte Kaverne. Da und dort im Oberlappen sind grössere und kleinere, tuberkulös pneumonische Herde zu erkennen. Daneben sind auch frische, bis linsengrosse Tuberkelknötchen nachweisbar. Schwielenbildungen fehlen im Oberlappen vollkommen.

Das Gewebe des Unterlappens erscheint im ganzen lufthaltig, ziemlich stark ödematös. Über das Gewebe verstreut finden sich vereinzelte schiefrig indurierte Tuberkelknötchen. Ausserdem ist der Unterlappen von einer grösseren Anzahl tuberkulös pneumonischer Herde, die zum Teil im Zentrum geringe Neigung zur Verkäsung erkennen lassen, durchsetzt. An den grösseren Gefässen und Bronchialstämmen sind hier keine auffälligen Veränderungen zu erkennen. Auch im Lungengewebe fehlen Schwielenbildungen vollkommen.

Mikroskopische Untersuchung. (Stück aus dem Mittellappen der rechten Lunge.)

Das Lungengewebe erscheint atelektatisch und atrophisch. Die Pleura ist in eine mehrere Millimeter dicke, fibröse Schwiele umgewandelt, deren Oberfläche von einem zellreichen, tuberkulösen Granulationsgewebe bedeckt ist. Die tieferen Schichten des Granulationsgewebes weisen zum Teil ausgedehntere regressive Veränderungen auf, während sich an der Oberfläche frischere, hier und da knötchenförmige Wucherungen erkennen lassen, welche durch zahlreiche Riesenzellen charakterisiert sind. Das ganze Bild entspricht einem. fortschreitenden tuberkulösen Prozesse der Pleura. Nur hier und. da ist eine geringe T'endenz zur Organisation in den tieferen Schichten der Pleuraauflagerung festzustellen. An den betreffenden Stellen ziehen feine fibrilläre Stränge in das Granulationsgewebe hinein. Das ganze atelektatische Lungengewebe ist netzartig durchzogen von breiten, derben Bindegewebszügen, die in ihrem Verlaufe den interlobulären Septen entsprechen und das Lungengewebe in einzelne Lobuli teilen. Die Bindegewebsschwielen nehmen ihren Ausgang rom gewucherten pleuralen Bindegewebe und senken sich in die breiten perivaskulären und peribronchialen Narben ein. Grössere und kleinere Gefässstämme und Bronchien zeigen weit klaffende Lumina und sind umgeben von einem breiten Wall derben, pigmentarmen Bindegewebes, welches als der Ausdruck einer abgeheilten Perivaskulitis und Peribronchitis erscheint. Das Lungengewebe selbst zeigt eine gleichmässige tuberkulös pneumonische Infiltration. Die Wände der Alveolen sind atrophisch, nur stellenweise bindegewebig verdickt. In den Alveolen liegen reichlich desquammierte Epithelien, Leukozyten und Ödemflüssigkeit. Die pneumonisch infiltrierten Herde zeigen in allen 
Partien eine mehr oder minder stark ausgeprägte Organisation durch junges fibroblastenreiches Bindegewebe. Das organisierende Bindegewebe enthält nur an vereinzelten Stellen geringe Mengen von Pigment. Neben den pneumonischen Herden finden sich Tuberkelknötchen in mässiger Zahl vor; sie sind zum Teil im Zentrum verkäst und ringsum von einem Wall jungen Bindegewebes, nach Art einer Kapsel, gegen das umgebende Lungengewebe abgeschlossen. Die Bindegewebskapsel selbst ist frei vom Granulationsgewebe, auch sind in direkter Umgebung keine progredienten Prozesse festzustellen. Doch fehlen die frischen tuberkulösen Prozesse im übrigen Lungengewebe keineswegs vollkommen.

Besonders auffallend ist die Bindegewebsentwickelung im Oberlappen. Entsprechend dem makroskopischen Befunde zeigt sich hier das respiratorische Parenchym stark reduziert, der grösste Teil des Oberlappengewebes ist in eine derbe, pigmentarme Bindegewebsschwiele umgewandelt. Frischere Prozesse fehlen hier vollkommen.

Das mikroskopische Bild des Unterlappens wird von pneumonischen und knötchenförmigen Tuberkelherden beherrscht. Die knötchenförmigen Prozesse zeigen vielfach zentrale Verkäsung; in den Randpartien besteht junges Granulationsgewebe mit reichlichen Riesenzellenbildungen. Nur ganz vereinzelt findet sich eine Abkapselung eines tuberkulösen Herdes. Überhaupt tritt die Bindegewebsentwickelung im Unterlappen gegenüber den fortschreitenden Prozessen auffallend in den Hintergrund. Nur in der Umgebung einzelner Gefässe und Bronchien bestehen stärkere Bindegewebsanhäufungen.

Das Gewebe der linken, nicht komprimierten Lunge enthält zahlreiche tuberkulös pneumonische Herde, an denen eine Neigung zur fibrösen Organisation nicht zu erkennen ist. Zwar fehlt auch hier eine geringe Bindegewebsentwickelung in der Umgebung von einzelnen Gefässen und Bronchien nicht, doch bleibt sie an Stärke noch weit hinter der Bindegewebsentwickelung des rechten, schwach komprimierten Unterlappens zurück.

Fasse ich das Resultat der mikroskopischen Untersuchung meiner drei Fälle noch einmal kurz zusammen, so ergibt sich daraus obne Zweifel, dass die komprimierte Lungə hochgradige Veränderungen erfahren hat. In der Hauptsache zeigt das Lungengewebe das histologische Bild atelektatischer Bezirke einer tuberkulösen Lunge. Auffallend ist dabei jedoch die ungewohnt starke Neigung des tuberkulösen Gewebes zur Schwielenbildung. Sowohl in der Umgebung von 
Gefässen und Bronchien hat eine ausgedehnte Entwickelung bindegewebiger Schwielen stattgefunden, die als der Ausdruck abgeheilter perivaskulärer und peribronchialer Entzündungsprozesse angesprochen werden muss; ausserdem zeigen auch die pneumonischen Herde und namentlich ältere verkäste Einzeltuberkel eine ausgesprochene narbige Umwandlung und Abkapselung gegen das umgebende Gewebe. Allerdings kann die Abheilung trotz der trockenen Verkäsung, wie sie namentlich bei den Herden in Fall II besteht und trotz des Fehlens von Epitheloidzellen und sonstigen Anzeichen frischer Prozesse keineswegs als vollkommen gelten. Aus dem Vorhandensein von tuberkulösem Granulationsgewebe innerhalb der bindegewebigen Kapseln selbst, sowie in deren direkten Umgebung erhellt zur Crenüge, dass zwar bei grösseren und kleineren tuberkulösen Herden eine ausgesprochene Tendenz zu bindegewebiger Ausheilung besteht, dass aber der infektiöse Prozess zwar zu einem gewissen Stillstand gekommen ist, wäbrend eine völlige Ausheilung noch nicht eingetreten ist. Für das Fortschreiten des Prozesses sprechen auch die allerdings nur wenigen, da und dort im Gewebe zerstreuten, frischen tuberkulösen Herde. Frische und ältere tuberkulöse Herde sind durch einen äusserst geringen Gehalt an Tuberkelbazillen charakterisiert; dabei erscheinen die frischen Tuberkel klein, riesenzellenreich, frei von Verkäsung, kurz, sie bieten das Bild, wie wir es so häufig in den Organen wenig disponierter Individuen beobachten können.

Wenn es nach den vorliegenden Befunden zu entscheiden gilt, ob sich in den komprimierten Lungen Veränderungen zeigen, die als eine Ausheilung tuberkulöser Prozesse gedeutet werden können, so unterliegt es meines Erachtens keinem Zweifel, dass die anatomischen Veränderungen der Lungen als Zeichen eines zum Stillstand gekommenen, in Ausheilung begriffenen tuberkulösen Prozesses gedeutet werden müssen.

Wesentlich schwieriger liegen die Verhältnisse für die Entscheidung, ob und inwieweit der künstliche Pneumothorax als Ursache für die Heilungsvorgänge verantwortlich gemacht werden kann. Das gleichmässige Vorkommen ausgedehnter Vernarbungsprozesse in allen drei Fällen spricht meines Erachtens ohne weiteres für einen ätiologischen Zusammenhang zwischen Bindegewebsentwickelung und Lungenkompression und zwar um so mehr, als die tuberkulösen Herde der atmenden Lunge eine ähnliche Neigung zur Vernarbung vermissen lassen. Allerdings lässt sich nicht in Abrede stellen, dass ein gewisses Missverbältnis zwischen der Stärke der Bindegewebsentwickelung und der Dauer der Kompressionsbehandlung besteht. Während im II. und III. Falle, bei einer etwa zweimonatlichen Kompressions- 
behandlung eine ausgedehnte Narbenbildung besteht, ist es im Falle I trotz des sieben Monate wirkenden Pneumothorax nur zu relativ geringen Heilungsprozessen gekommen. Dieser Widerspruch findet seine Erklärung im klinischen Verlaufe des I. Falles (Mischinfektion mit Staphylokokken). Hier lag bereits zur Zeit der Anlegung des künstlichen Pneumothorax eine so hochgradige Herabsetzung der gesamten Resistenzfähigkeit des Organismus vor, dass eine ausreichende lokale Reaktion von vorneherein nicht zu erwarten war. Dazu kommt noch die Tatsache, dass infolge der ausgedehnten Verwachsungen ein ausreichender Pneumothorax, nach Forlanini die Grundbedingung für einen vollkommenen Erfolg, nicht zu erreichen gewesen war.

Forlanini (1) bat besonders neuerdings wieder darauf hingewiesen, wie sehr die Aussichten auf Ausheilung von der Vollständigkeit des Pneumothorax abhängig sind, eine Tatsache, die auch durch die histologischen Untersuchungen ihre Bestätigung findet. Vergleicht man die Intensität der Bindegewebsbildung in den einzelnen Lungenabschnitten, so lässt sich ohne Schwierigkeit eine gewisse Gesetzmässigkeit erkennen, indem das Narbengewebe um so stärker entwickelt ist, je weniger voluminös der betreffende Lungenabschnitt ist, $d . h$. je ausgiebiger der Druck an den einzelnen Stellen zur Wirkung gekommen ist. Man findet deshalb in den Randpartien der einzelnen Lungenlappen und namentlich in den Spitzen der Oberlappen vorwiegend starke Narbenbildungen, während in den zentral gelegenen Lungenabschnitten die Schwielenbildung weniger stark entwickelt ist. In Partien, in denen infolge starker Verwachsungen der Pneumothoraxdruck nur zum Teil zur Wirkung gelangen konnte, tritt die Bindegewebsentwickelung stark in den Hintergrund, wie dies namentlich die Untersuchung der ausgedehnt verwachsenen Unterlappen in Fall III deutlich nachweisen liess.

Die weitaus günstigsten Verhältnisse zeigt Fall II. Verantwortlich sind hier für den Erfolg zwei Faktoren zu machen. Durch das Fehlen ausgedehnterer Verwachsungen hat hier der Pneumothorax am vollständigsten die Bedingungen hinsichtlich des notwendigen Volumens und Druckes erfültt. Dazu kommt in Fall II noch die Natur des tuberkulösen Prozesses. Während in Fall I und III die pneumonischen Prozesse vorherrschen, finden sich im II. Fall vorwiegend herdförmige Prozesse. Die Beobachtung Forlaninis, dass akut verlaufende pneumonische Prozesse für die Kompressionsbehandlung ungeeignet und der Pneumothorax hierbei geradezu kontraindiziert sei, findet in den histologischen Verhältnissen eine gewisse Stütze. Es liegt nach den ganzen Bildern klar, dass die sich langsamer ent- 
wickelnden Herderkrankungen für die Narbenbildungen ein günstigeres Objekt darstellen, als die progredienten pneumonischen Prozesse.

Vor allem möchte ich nun dem Einwand begegnen, dass die histologischen Veränderungen des Lungengewebes, welche meines Erachtens als der Effekt der Kompressionsbehandlung angesehen werden müssen, schon vor Anlegung des Pneumothorax bestanden haben konnten, dass es sich also lediglich um eine sehr ausgeprägte Form der indurierenden Phthise handle. Ich möchte demgegenüber nochmals betonen, dass es jedenfalls mehr als ein Zufall ist, dass sich in den drei nach gleicher Methode behandelten Fällen, in der komprimierten Lunge ausgesprochene Vernarbung tuberkulöser Prozesse findet, während die atmende Lunge keine narbige Veränderung ihrer tuberkulösen Herde aufweist. Dazu kommt noch, dass es sich klinisch wie anatomisch keineswegs um chronische Prozesse handelt, wie man sie häufig bei alten Phthisikern beobachtet. Namentlich das histologische Bild der tuberkulösen Herde lässt überall die Neigung auch frischerer Tuberkelknötchen zur fibrösen Umwandung vermissen. Dazı fehlt in den Bindegewebsschwielen das Pigment so gut wie vollkommen, ein sicheres Zeichen, dass es sich nicht um alte Schwielen handeln kann, da sich gerade in ihnen mit Vorliebe das Pigment bei chronischer Lungenphthise ablagert.

In welcher Weise hat man sich wohl die Wirkung des Pneumothorax auf die tuberkulöse Lunge zu erklären?

Zwei Faktoren sind es, die vorwiegend durch die Kompression der Lunge beeinflusst werden: Atmung und Zirkulation, meines Erachtens die beiden Hauptwege für die Weiterverbreitung des tuberkulösen Prozesses innerhalb der Lungen. Solange die erkrankte Lunge sich in Tätigkeit befindet, erfolgt ein Fortschreiten des Prozesses, indem durch Aspiration Alveole um Alveole in den Krankheitsherd mit einbezogen wird. Durch die Kompression des Lungengewebes wird dann eine Entspannung bedingt, wodurch die zahlreichen, mit infektiösen Massen gefüllten Hohlräume, die eine ständige Quelle für die Weiterscbleppung des ganzen Prozesses bieten, ausgeschaltet werden. Die tuberkulösen Massen bleiben dann an Ort und Stelle liegen und erfahren hier das Schicksal der Fremdkörper, die Abkapselung durch Bindegewebe.

Die Hauptwirkung des Pneumothorax wird von einigen Autoren den veränderten Zirkulationsverhältnissen zugeschrieben. Namentlich Czerniki führt die Wirkung auf eine durch die Kompression bedingte Anämie zurück. Nicht nur die Sekretion der Bronchialschleimhaut und der Kavernen soll dadurch abgeschwächt werden, sondern letztere sogar dadurch zum Schwund kommen. Brauer 
vermutet dagegen, dass es sich vielleicht um eine Wirkung der Blutstauung im Sinne Bi ers handeln könne. Neuerdings neigt B r a u er(12) allerdings mehr dazu, die Heilungsvorgänge auf die Verlangsamung der Lymphzirkulation zurückzuführen. Ich schliesse mich dieser letzten Auffassung Brauers an. Durch die Ausschaltung der Atmung, die einen wesentlichen Faktor für eine regelmässige Lymphzirkulation darstellt, muss naturgemäss eine Stauung der Lymphe eintreten. Durch diese Lymphstauung wird eine Resorption von Toxinen in den Organismus wesentlich herabgesetzt. In dieser verminderten Toxinresorption findet auch die auffallend günstige Beeinflussung des Allgemeinbefindens (Herabsetzung der Temperatur) eine ausreichende Erklärung. Besonders beachtenswert erscheinen mir für die Bewertung der verminderten Toxinresorption die Bestimmungen des "opsonischen Index" bei künstlichem Pneumothorax durch Pigger (13). Die dauernde Erhöhung des opsonischen Index bei Ruhigstellung der tuberkulösen Lunge in allen von Pigger untersuchten Fällen lässt sich zwanglos als die Folge einer verminderten Toxinresorption deuten. Nach Wrights eigenen Feststellungen ist bekanntlich die Erhöhung des „opsonic Index" als ein Zeichen erhöhter Resistenz des gesamten Organismus anzusehen. Hieraus erklärt sich dann auch ohne weiteres die erhöhte Reaktionsfähigkeit des Organismus gegenüber lokalisierten Prozessen. In der verminderten Resorption der Proteine ist also der Hauptfaktor für die Ausheilung des tuberkulösen Prozesses zu suchen. Die Bindegewebsentwickelung hat demnach als eine verstärkte reaktive Erscheinung zu gelten, die ihren Grund in der Frhöhung der Resistenz des Gesamtorganismus und in dem vermehrten lokalen Reiz der tuberkulösen Toxine hat.

Atmungs- und Zirkulationsverhältnisse beim künstlichen Pneumothorax sind der Gegenstand eingehender experimenteller Untersuchungen gewesen, die Herr Prof. Brauer in seiner Klinik durch den Japaner Herrn Shingu hat vornehmen lassen. Bei der prinzipiellen Bedeutung dieser Versuche möchte ich es nicht unterlassen, kurz auf dieselben einzugehen. Es handelt sich dabei um zwei Versuchsreihen an Kaninchen.

Erster Versuch: Anlegung eines künstlichen Pneumothorax, dann Aspiration von Russ: die pathologische Lunge bleibt frei von Russ.

Zweiter Versuch: Aspiration von Russ, dann Pneumothorax: die pathologische Lunge bleibt länger russhaltig als die atmende. In letzterer liegen die Russpartikelchen vorwiegend in den Alveolen und Bronchiolen, meist in Zellen eingeschlossen. In der Pneumothoraxlunge sind die russhaltigen Zellen reichlich in das Bindegewebe eingedrungen und füllen auch die kleinen endopulmonalen Lymph- 
drüsen, sowie die Bronchialdrüsen am Hilus stärker als bei der atmenden Lunge.

Das Resümee der Versuche ist nach Beneke folgendes: „Die Atmung befördert die Inspiration von Fremdkörpern, erleichtert aber auch die Exspiration derselben. Sehr wahrscheinlich hat dies Verhältnis auch für bakterienbeladene Fremdkörper eine Bedeutung. - Die Atelektase erleichtert das Eindringen von Fremdkörpern in das Saftspaltensystem der Lunge. Etwaige Bakterien werden auf diese Weise dem Körper reichlicher zugeführt, - aber gleichzeitig vielleicht dadurch auch eher unschädlich gemacht, falls die Säfte und Zellen des Organismus bakterizid sind.

Sind bereits tuberkulöse Herde in einer Lunge vorhanden, so hemmt die nachträgliche Atelektase sicher die Lymphbewegung und damit die rasche Verbreitung der Baktericn und erleichtert die reaktiven Gewebswucherungen."

Auch Beneke nimmt demnach auf Grund dieser experimentellen Untersuchungen eine günstige Beeinflussung des tuberkulösen Prozesses an und sieht ebenfalls den wesentlichen Faktor für die reaktiven Gewebswucherungen in Veränderungen der Lymphzirkulation. Dabei darf allerdings nicht vergessen werden, dass gerade in dem leichten Eindringen von Fremdkörpern in die Saftspalten eine Gefahr einer Verallgemeiernung des Prozesses im Organismus gegeben ist. Ich habe angesichts der Versuche Shingus besonders bei der Untersuchung der bronchialen Lymphdrüsen darauf geachtet, ob etwa in ihnen eine Zunahme des tuberkulösen Prozesses, namentlich eine grössere Menge frischer Tuberkeln nachzuweisen seien, konnte aber keinerlei Anhaltspunkte dafür gewinnen. Es handelt sich durchweg um ältere, verkäste Herde; frische Tuberkeln fehlen so gut wie ganz.

Zum Schluss möchte ich es nicht unterlassen, noch auf einige, meines Erachtens keineswegs gering zu schätzende Komplikationen hinzuweisen, die aus der Anlegung des künstlichen Pneumothorax sich ergeben können, ich meine die Perforation käsiger Herde in den Pleuraraum und die Entstehung ausgedehnter tuberkulöser Pleuritiden. Die knöcherne Thoraxwand bildet einen Schutz gegen den Durchbruch der Käseherde durch die Möglichkeit pleuritischer Verwachsungen. Sobald die Lunge kollabiert und des natürlichen Widerstandes entbehrt, gelangen die Käseherde ohne Schwierigkeit bis an die Pleura und führen mit ihrem Durchbruch die auch vom Kliniker so sehr gefürchtete Komplikation der eitrigen Pleuritis herbei. 
Die Gefahr ausgedehnter Pleuritiden liegt in der Produktion grösserer Mengen Tuberkelbazillen im Pleuraraum und in der gesteigerten Toxinresorption. Ausserdem können auch rasch ansteigende Pleuraexsudate, falls sie nicht durch Punktion entfernt werden, einen ungünstigen Ausgang herbeiführen, wie dies in Fall II nach Brauers

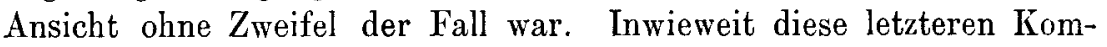
plikationen in der Lage sind, den heilenden Einfluss des Lungenkollapses $\mathrm{zu}$ beeinträchtigen, darïber müssen weitere klinische Beobachtungen entscheiden.

Zusammenfassung.

Die vorliegenden Untersuchungen berechtigen meines Erachtens zu folgenden Schlüssen:

1. Die Ruhigstellung der tuberkulösen Lunge bedingt einen Stillstand des tuberkulösen Prozesses mit anschliessender Ausheilung.

2. Die Ausheilung tritt in der Abkapselung käsiger Herde und Organisation pneumonischer Prozesse durch Bindegewebe zutage.

3. Die reaktive Bindegewebswucherung hat ihren Grund in der Verlangsamung der Lymphkzirkulation und der dadurch bedingten verminderten Resorption der tuberkulösen Toxine.

4. Das Resultat der Kompressionsbehandlung ist abhängig ron der Vollständigkeit des Pneumothorax und von einem genügend starken, zunächst eine Entfaltung der Lunge ausschliessenden Druck.

5. Die pneumonische, stark progrediente Form der Lungentuberkulose erscheint für die Kompressionsbehandlung weniger geeignet als die knötchenförmigen Prozesse.

6. Die Atelektase der Lunge begünstigt, wie dies experimentell erwiesen ist, das Eindringen von Fremdkörpern in die Lymphbahnen. Hierin könnte eine Gefahr des Pneumothorax bestehen; die Verschleppung des tuberkulösen Prozesses von der erkrankten Lunge aus zunächst in die Bronchiallymphdrüsen und ron dort in den übrigen Organismus. In meinen Fällen liess sich eine solche Verschleppung in die Lymphdrüsen allerdings nicht beobachten. Die klinischen Erfahrungen (Brauer) scheinen der Annahme der Verschleppung zu widersprechen.

7. Perforation käsiger Herde und ausgedehnte Pleuritiden stellen gefährliche Komplikationen des Pneumothorax dar.

Zum Schlusse erlaube ich mir, Herrn Professor Bra uer für die liebenswürdige C̈berlassung des Materials, sowie meinem hochverehrten Chef, Herrn Professor Beneke, für die freundlichen Ratschläge bei der Ausarbeitung dieser Untersuchungen meinen ergebensten Dank auszusprechen. 


\section{Literatur.}

1. Forlanini, Zur Behandlung der Lungenschwindsucht durch künstlich erzeugten Pneumothorax. D. med. W. 1906.

2. Späth, Beziehungen der Lungenkompression zur Lungentuberkulose. Württemb. Korrespondenzbl. Bd. 58. Nr. 15.

3. Bäumler, Die Behandlung der Pleuraempyeme bei an Lungentuberkulose Loidenden. D. med. W. Nr. 37 u. 38. 1894.

4. Spengler, L., Über mehrere Fälle von geheilten tuberkulösen Pneumothorax mit gleichzeitiger Heilung der T'uberkulose. Zeitschr. f. Tuberkulose u. Heilstättenwesen 1901. Bd. II. H. I und II.

5. Derselbe, Zur Chirurgie des Pneumothorax. Mitteilung über zebn eigene Fälle von geheiltem tuberkulösem Pneumothorax, verbunden in sechs Fällen mit gleichzeitiger Heilung der Lungentuberkulose. Beiträge z. klin. Chirurgie. Bd. 49.

6. Gaillard, Zitiert nach Mosheim.

7. Adams, F. E., Zitiert nach Mosheim.

8. Murphy, Surgery of the lung. Journ. Amer. Assoc. July u. August 1898.

8a. Liemke, Zitiert nach Mosheim.

9. Brauer, L., Über Pneumothorax. Marburg 1906.

10. Derselbe, Der therapeutische Pneumothorax. D. med. W. 1906. Nr. 35.

11. Derselbe, Die Behandlung chronischer Lungenkrankheiten durch Lungenkollaps. Therapie der Gegenwart. Juni 1908.

12. Derselbe, Kongress für innere Medizin, Wien 1908.

13. Ders elbe, Kongress für Chirurgie, Berlin 1908.

14. Drasche, Über die operative Behandlung des tuberkulösen Pneumothorax. Wiener klin. W. Nr. 45 und 46. 1899.

15. Mosheim, Die Heilungsaussichten der Lungentuberkulose bei spontanem und künstlichem Pneumothorax. Beitr. z. Klinik der Tuberkulose. H. III.

\section{Erklärung der Abbildungen.}

Die Abbildungen sind nach den Originalpräparaten hergestellt, welche der mikroskopischen Beschreibung zugrunde liegen. (Färbung nach van Gieson, Bindegewebe leuchtend rat.)

Fig. 1 und 2. Schnitte aus zwei verschiedenen Stellen der stark komprimierten Randpartien des Unterlappens (Fall II) : Abkapselung verkäster Tuberkelherde, Entwickelung starker perivaskulärer und peribronchialer Bindegewebsschwielen.

Hierzu Fig. 6. Teil von Fig. 1 bei stärkerer Vergrösserung: Zwei grosse, käsige Herde, ringsum durch Bindegewebe abgekapselt. 
Fig. 3. Schnitt aus zentral gelegenen Partien des unteren Lappens; nur geringe Bindege websentwickelung.

Fig. 4. Schnitt aus dem freien Rand des Oberlappens (Fall III): Das atelektatische Lungengewebe netzförmig von Bindegewebsschwielen durchzogen.

Fig. 5. Schnitt aus der Spitze des Oberlappens: Sehr starke Entwickelung von Bindegewebsschwielen.

Fig. 7. Schnitt aus dem nur wenig komprimierten unteren Lappen (Fall III): Bindegewebe nur mässig stark entwickelt.

Die Zeichnungen sind ausser Fig. 6 mit der Lupe gezeichnet. 
Fig. 1.

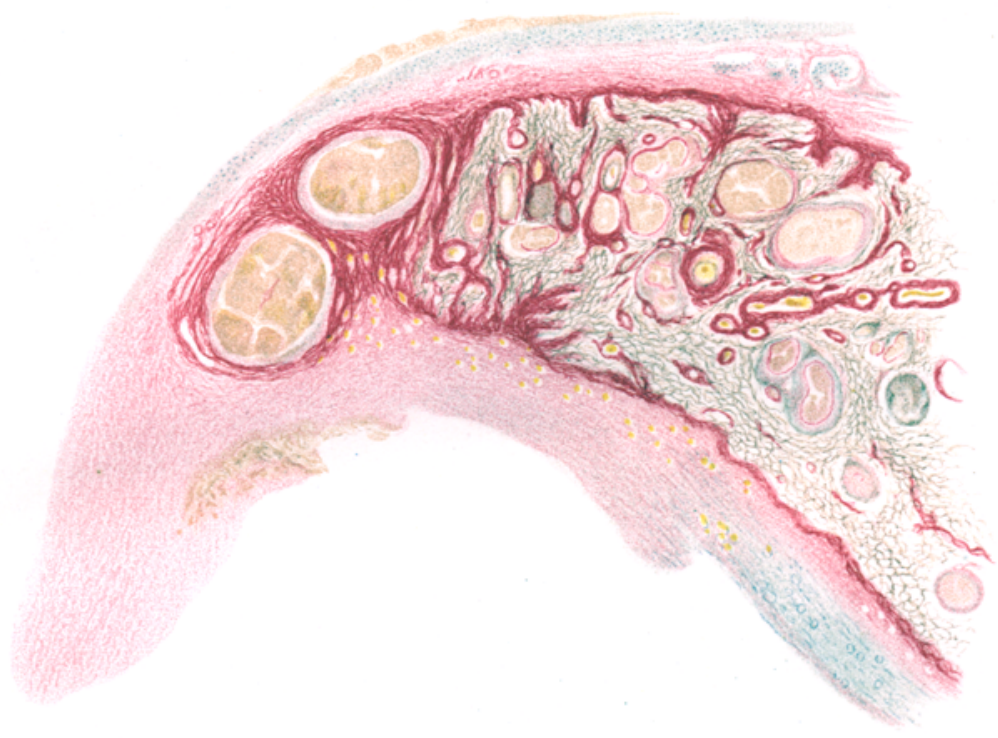

Fig. 2.

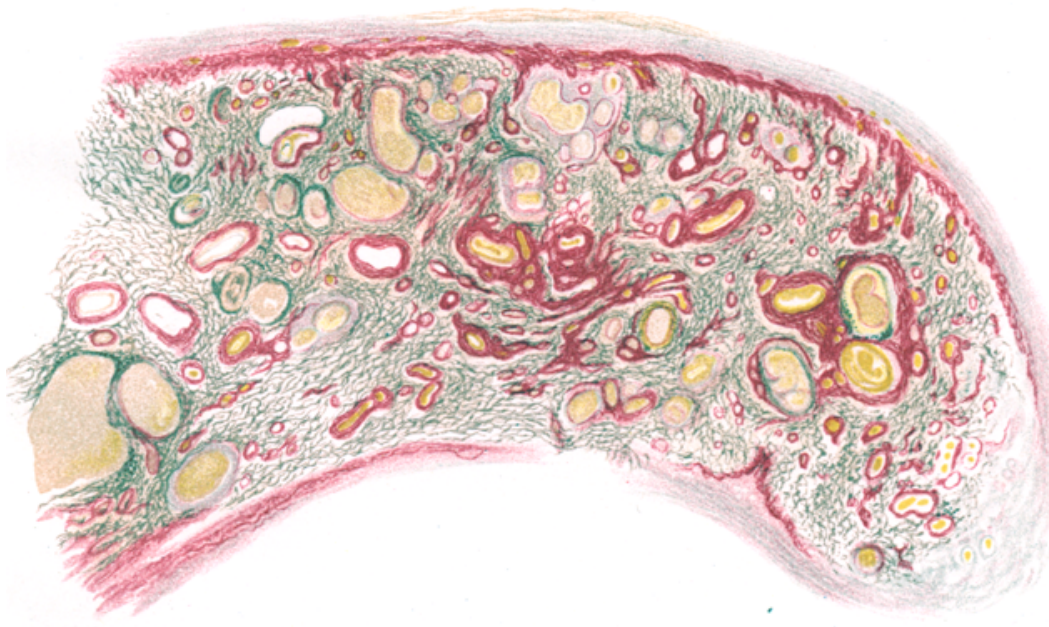


Fig. 3.

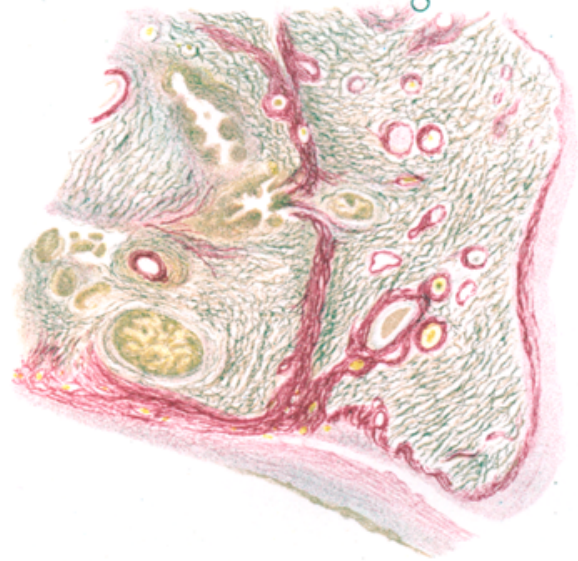

Fig. 4 .

Fig. 5.
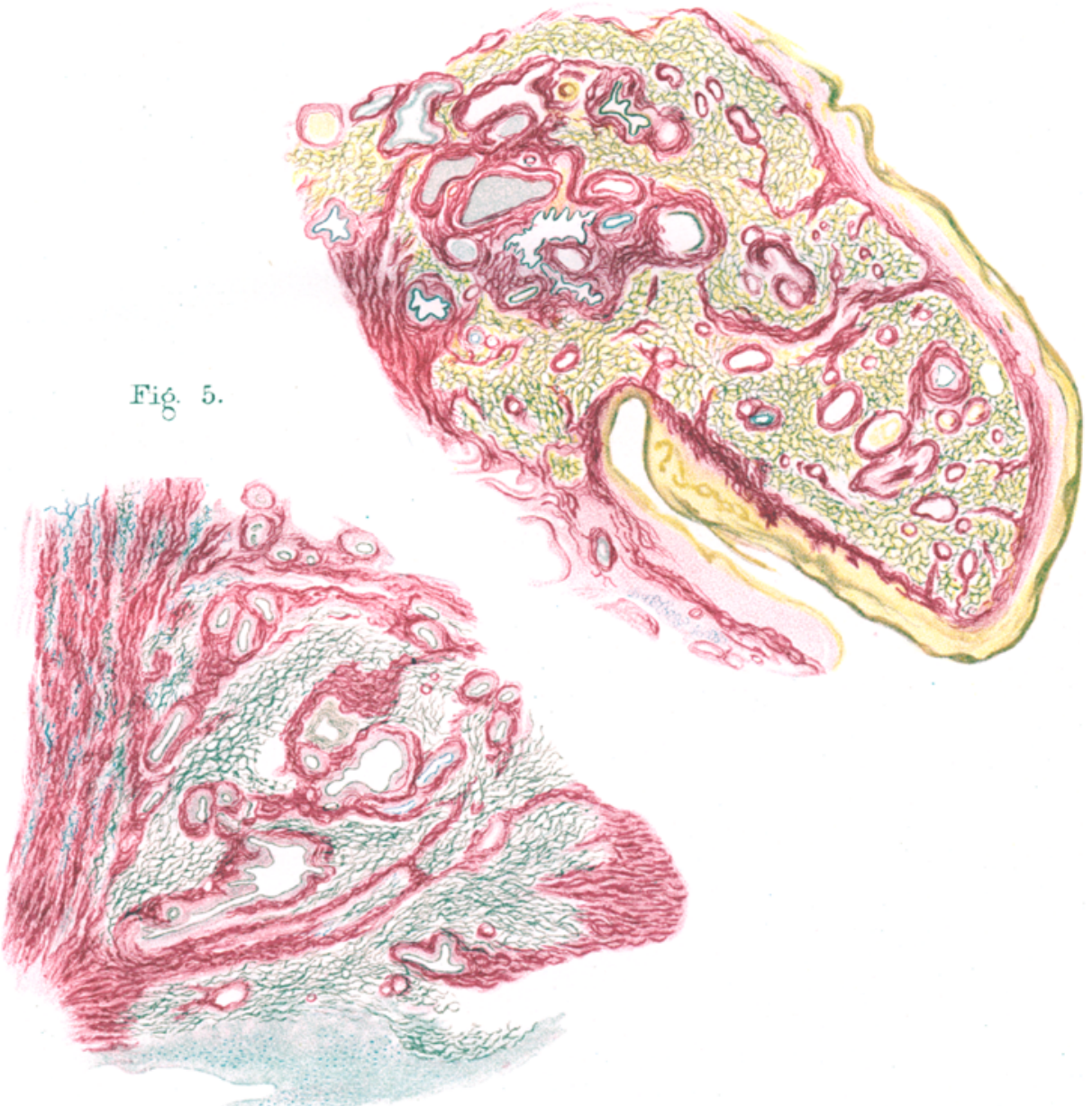

Gmefz, Der Finfluss des künstlichen Ihrumothornx: ouf lie tuberkulöse launge. 
Fig. 6 .

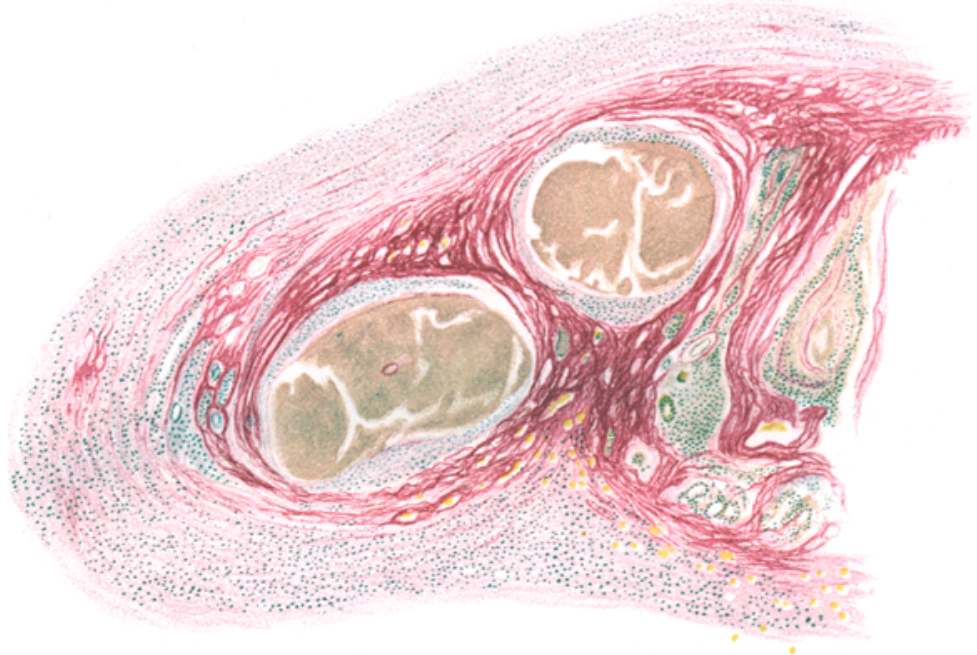

Fig. 7.

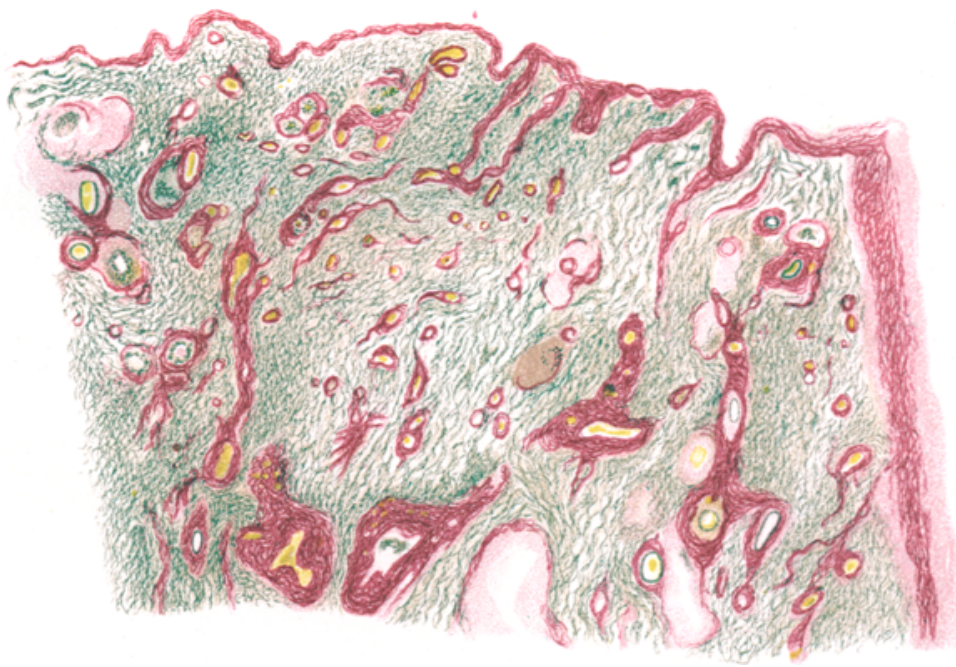

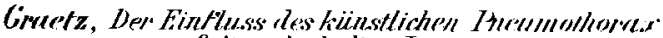
ruf die lubrrkuläse Lunge. 